\title{
CHARACTERISATIONS OF GENERALISED UNISERIAL ALGEBRAS. III
}

\author{
by DRURY W. WALL \\ (Received 19th October 1964)
}

\section{Introduction}

Let $A$ be a finite dimensional algebra with identity element over a field. $A$ is generalised uniserial if every primitive left ideal and every primitive right ideal of $A$ has only one compositions series. In the previous papers in this series $(6,7)$ generalised uniserial algebras have been characterised as algebras all of whose residue class algebras are of certain types. The purpose of this paper is to extend the earlier results by showing that in order that $A$ be generalised uniserial it is sufficient to require weaker conditions on merely a finite sequence of residue class algebras of $A$.

A primitive ideal $A e$ of $A$ is called dominant if it is dual to some primitive ideal $f A$. In a quasi-Frobenius algebra every primitive ideal is dominant and an algebra is uniserial if all of its residue class algebras are quasi-Frobenius (6). There are a number of types of algebras, more general than the quasiFrobenius, in which dominant ideals exist and in which every primitive ideal is related in some way to the dominant ideals. (For details see (5) and (8).) It is known (7) that an algebra $A$ is generalised uniserial if and only if every residue class algebra of $A$ has a unique minimal faithful representation or, equivalently, is a $Q F-3$, algebra (one in which every primitive ideal is weakly subordinate to a set of dominant ideals).

$\S 2$ contains the definitions and notations for the paper while in $\S 3$ is constructed a sequence of residue class algebras $A_{i}$ of $A$ by using the socles of the dominant parts of the algebras. An algebra $A$ is called dominant if there exist dominant ideals in $A . \$ 4$ contains the main result of the paper, namely, that $A$ is generalised uniserial if every $A_{i}$ is a dominant algebra. In $\S 5$ is given an example to show that in order for $A$ to be generalised uniserial it is not sufficient that every $A / N^{i}$ be dominant, where $N=\operatorname{Rad} A$.

\section{Definitions and notations}

Let $A$ be a finite dimensional algebra with identity element 1 over a field. Let

$$
1=\sum_{i=1}^{n} \sum_{j=1}^{f_{i}} e_{i j}
$$

be a decomposition of the identity element into the sum of mutually orthogonal 
primitive idempotents such that $e_{i j} \cong e_{h k}$ if and only if $i=h$. If $e$ and $f$ are idempotents then $e \cong f$ if and only if $A e \cong A f$ (or equivalently $e A \cong f A$ ). For $i=1, \ldots, n$ denote $e_{i 1}$ by $e_{i}$ and let $E_{i}=\sum_{j=1}^{f_{1}} e_{i j}$. If $e$ is any primitive idempotent of $A$ then the left ideal $A e$ is called dominant if there exists a primitive idempotent $f$ such that $A e$ is dual to $f A$. If $A$ has at least one dominant left ideal (and hence at least one dominant right ideal) then $A$ is called dominant.

As in a previous paper (9), for a given algebra $A$ and a fixed decomposition (1) let $\Sigma=\left\{i \mid A e_{i}\right.$ is dominant $\}$ and let $\Pi=\left\{i \mid e_{i} A\right.$ is dominant $\}$. For these choices of $A$ and (1) let

and

$$
D(A)=\sum_{i \in \Sigma} \sum_{j=1}^{f_{1}} A e_{i j}
$$

$$
D^{\prime}(A)=\sum_{i \in \Pi} \sum_{j=1}^{f_{i}} e_{i j} A .
$$

$D(A)$ is called the left dominant part and $D^{\prime}(A)$ the right dominant part of $A$ with respect to the decomposition (1).

Every primitive left ideal $A e$ is isomorphic to one and only one of the $A e_{i}$ and every primitive right ideal is isomorphic to one and only one of the $e_{i} A$. Thus, if $A$ has a dominant left ideal $A e$ then there is some $i$ such that $A e_{i}$ is dominant and hence $i \in \Sigma$. Hence, in the present notation, each of the following is equivalent to $A$ being quasi-Frobenius: $\Sigma=\{1, \ldots, n\}$, $\Pi=\{1, \ldots, n\}, D(A)=A$, or $D^{\prime}(A)=A$. Also, $A$ is dominant if and only if one of the following hold: $\Sigma$ is not empty, $\Pi$ is not empty, $D(A) \neq 0$, or $D^{\prime}(A) \neq 0$. (We adopt the convention that $D(A)=0$ if $\Sigma$ is the empty set.)

In the case when $A$ is dominant but not quasi-Frobenius the sets $\Sigma$ and $\Pi$ need not be equal but they must have the same order. For each $i \in \Sigma$ let $\rho(i)$ be the unique integer such that $A e_{i}$ is dual to $e_{\rho(i)} A$. Then $\rho: \Sigma \rightarrow \Pi$ is a bijection.

For any left $A$-module $M$ let $S(M)$ denote the $A$-socle of $M$, i.e., the sum of all simple $A$-submodules of $M$. Similarly let $S\left(M^{\prime}\right)$ denote the $A$-socle of a right $A$-module $M^{\prime}$. It is known (5) that if $i \varepsilon \Sigma$ then $A e_{i}$ (and hence each $\left.A e_{i j}, j=1, \ldots, f_{i}\right)$ has only one minimal subideal and therefore $S\left(A e_{i}\right)$ is simple as a left ideal.

From the properties of socles (see (2, p. 63), (8, §5)) and equations (2) and (3) it follows that

and

$$
S(D(A))=\sum_{i \in \Sigma} \sum_{j=1}^{f_{k}} S\left(A e_{i j}\right)
$$

$$
S\left(D^{\prime}(A)\right)=\sum_{i \in \Pi} \sum_{j=1}^{f_{l}} S\left(e_{i j} A\right)
$$

and both sums are direct, (4) as a sum of left $A$-modules and (5) as a sum of right $A$-modules. 
By applying earlier results, principally Lemmas 4 and 5 of (6), we obtain the following: (a) for each $i \in \Sigma, S\left(A e_{i}\right) A$ is a two-sided ideal and

and

$$
S\left(A e_{i}\right) A=\sum_{j=1}^{f_{1}} S\left(A e_{i j}\right)=U\left\{S(A f) \mid A f A \cong e_{i}\right\}
$$

$$
S\left(A e_{i}\right) A=A S\left(e_{\rho(i)} A\right)
$$

(b) $S(D(A))$ is a two-sided ideal of $A$ and

$$
S(D(A))=S\left(D^{\prime}(A)\right) \text {. }
$$

To prove (8), we use (4), (6), (7), the right dual of (6) and (5) as follows:

$$
\begin{aligned}
S(D(A))=\sum_{i \in \Sigma} \sum_{j=1}^{f_{l}} S\left(A e_{i j}\right)=\sum_{i \in \Sigma} S\left(A e_{i}\right) A \\
\quad=\sum_{i \in \Sigma} A S\left(e_{\rho(i)} A\right)=\sum_{i \in \Pi} \sum_{j=1}^{f_{i}} S\left(e_{i j} A\right)=S\left(D^{\prime}(A)\right) .
\end{aligned}
$$

Thus, even though $D(A)$ and $D^{\prime}(A)$ need not be equal their socles are. Also (6) shows that $S(D(A))$ is independent of the choice of the decomposition (1).

\section{The sequence $\left\{A_{i}\right\}$}

Definition 1. For any $A$ let $A^{*}=A / S(D(A))$, i.e., $A^{*}$ is the residue class algebra of $A$ with respect to the two-sided ideal $S(D(A))$, which is the socle of the dominant part of $A$.

Definition 2. Let $\left\{A_{i}\right\}$ be the sequence defined inductively:

$$
A_{0}=A ; A_{i}=A_{i-1}^{*}=A_{i-1} / S\left(D\left(A_{i-1}\right)\right), i \geqq 1 .
$$

Definition 3. For each $i \geqq 1$ let $h_{i}: A_{i-1} \rightarrow A_{i}$ be the natural epimorphism with ker $h_{i}=S\left(D\left(A_{i-1}\right)\right)$. Let $g_{i}: A \rightarrow A_{i}$ be defined inductively:

$$
g_{1}=h_{1} ; g_{i}=h_{i} g_{i-1}, i \geqq 2 \text {. }
$$

Definition 4. Let the sequence $\left\{Z_{i}\right\}$ of two-sided ideals of $A$ be as follows:

$$
Z_{0}=\{0\} ; Z_{i}=\operatorname{ker} g_{i}, i \geqq 1 \text {. }
$$

Lemma 1. For each $i \geqq 1$,

$$
A / Z_{i} \cong A_{i}, Z_{i} / Z_{i-1} \cong S\left(D\left(A_{i-1}\right)\right),
$$

and $Z_{i} / Z_{i-1}$ is semisimple both as a left $A$-module and as a right $A$-module.

Proof. Omitted.

The sequence $\left\{Z_{i}\right\}$ is an ascending sequence of two-sided ideals of $A$ and therefore there exists a positive integer $m$ such that for all $i \geqq m$,

$$
Z_{i}=Z_{m} ; A_{i}=A_{m}, S\left(D\left(A_{i}\right)\right)=S\left(D\left(A_{m}\right)=\{0\} .\right.
$$

There are two possibilities that can occur, either $A_{m}=\{0\}$ or $A_{m}>\{0\}$.

Lemma 2. If $A_{m}>\{0\}$ then $A$ has a residue class algebra, namely $A / Z_{m}$, which is not dominant. 
Proof. If $A_{m}>\{0\}$ then since $S\left(D\left(A_{m}\right)\right)=\{0\}, A_{m}$ is a non-zero algebra with no dominant left or right ideals and hence $A_{m}$ is not a dominant algebra. But, by (12), $A / Z_{m} \cong A_{m}$ and thus, $Z_{m}$ is a proper two-sided ideal of $A$ such that $A / Z_{m}$ is not dominant.

\section{Characterisation theorem}

Theorem. If $A$ is not generalised uniserial then $A_{m}$ is not dominant.

Proof. If $A$ is not generalised uniserial there is a primitive idempotent $e$ such that either $A e$ or $e A$ has more than one composition series. Let us consider only the case where $A e$ has more than one composition series. Since, as noted in $\S 2$, there is some integer $i$ such that $A e \cong A e_{i}$, we shall assume that $e$ is chosen to be this $e_{i}$.

Let $L_{0}=\{0\}$. Then, $L_{0}$ is the trivial initial term in any composition series for $A e$. If $A e$ has only one minimal (non-zero) subideal let it be $L_{1}$. In this case, every composition series for $A e$ would contain $L_{0}$ and $L_{1}$ as the first and second terms. If for any integer $h, h \geqq 1$, there are subideals $L_{0}, L_{1}, \ldots, L_{h-1}$ of $A e$ which appear as the first $h$ terms in every composition series of $A e$ and if there is only one subideal $L$ of $A e$ such that $L_{h-1} \subset L$ and $L / L_{h-1}$ is a simple left $A$-module then let $L_{n}=L$. Since, $A e$ is assumed to have more than one composition series there is some smallest $h$ such that $L_{h}$ is not defined.

For this $h$ let $M=L_{h-1}$. Thus, there exist subideals $M^{\prime}$ and $M^{*}$ of $A e$ such that $M^{\prime} \neq M^{*}, M \subset M^{\prime}, M \subset M^{*}$ and both $M / M$ and $M^{*} / M$ are simple left $A$-modules.

(a) Assume that there is some integer $t$ such that $M \subset Z_{t}$ and consider the smallest such $t$. Let $t=k-1$. Then $M \subset Z_{k-1}$ and $M \not \subset Z_{k-2}$. Thus, from (11) and (12), it follows that

$$
g_{k-1}(M)=0, g_{k-2}(M) \neq 0, g_{k-2}(M) \subset g_{k-2}\left(Z_{k-1}\right) .
$$

But $g_{k-2}\left(Z_{k-1}\right)=Z_{k-1} / Z_{k-2}$ and is a semi-simple left $A$-module and therefore, by (14) $g_{k-2}(M)$ is also a semi-simple left $A$-module.

Since $e$ was chosen as one of the $e_{i}$ of the decomposition (1) and since the constructions of $\S 3$ are independent of (1), the results of Lemma 1 and equation (4) give

$$
\operatorname{Aen} Z_{k-1}=M
$$

and $A e / M=A e /\left(A e \cap Z_{k-1}\right)=g_{k-1}(A e) \cong A_{k-1} e^{\prime}$ where $e^{\prime}$ is a primitive idempotent of $A_{k-1}$.

But, considered as a primitive left ideal of $A_{k-1}, A e / M$ has more than one minimal subideal, i.e., both $M^{\prime} / M$ and $M^{*} / M$ are minimal subideals of $A e / M$ and hence neither $M^{\prime} / M$ nor $M^{*} / M$ can be contained in $S\left(D\left(A_{k-1}\right)\right)$. Thus,

$$
A e / M \cap S\left(D\left(A_{k-1}\right)\right)=\{0\} \text {. }
$$

Therefore, by Lemma 1 and (15), $\left[A e /\left(Z_{k-1} \cap A e\right)\right] \cap\left[Z_{k} / Z_{k-1}\right]=\{0\}$ and hence $\left[A e /\left(Z_{k-1} \cap A e\right)\right] \cap\left[\left(A e \cap Z_{k}\right) /\left(A e \cap Z_{k-1}\right)\right]=\{0\}$. Thus,

$$
\left(\operatorname{Aen} Z_{k}\right) /\left(\operatorname{Aen} Z_{k-1}\right)=\{0\}
$$


and

$$
\operatorname{Aen} Z_{k}=\operatorname{Aen} Z_{k-1} \text {. }
$$
$r>k$

Therefore $A e / M \cong g_{k-1}(A e) \cong g_{k}(A e)$. Inductively, it follows that for any

$$
g_{r}(A e) \cong g_{k}(A e) \cong A e / M
$$

Thus, in each $A_{r}$, for $r>k$, there is a left ideal isomorphic to $A e / M$ and hence $A_{r} \neq\{0\}$. In particular, the $A_{m}$ of (13) is not zero and hence $A_{m}$ is not dominant.

(b) Assume that there exists no $k$ such that $M \subset Z_{k}$. Then $Z_{m} \neq A$ and $A / Z_{m} \neq\{0\}$ and thus $A_{m} \neq\{0\}$ and $A_{m}$ is not dominant.

Corollary. $A$ is generalised uniserial if and only if for all $n, A / Z_{n}$ is a dominant algebra.

The corollary generalises the earlier result [7] that $A$ is generalised uniserial if and only if every residue class algebra of $A$ is $Q F-3(U M F R)$. An algebra is $Q F-3$ if every primitive ideal is weakly subordinate to a set of dominant ideals or, equivalently if it has a unique minimal faithful representation $(U M F R)$. Thus every $Q F-3$ algebra is a dominant algebra. However the converse is false.

\section{Example}

Let $A$ be the set of all matrices of the form in (19)

$$
\left(\begin{array}{lllll}
\alpha_{1} & 0 & 0 & 0 & 0 \\
\alpha_{5} & \alpha_{2} & 0 & 0 & 0 \\
0 & 0 & \alpha_{3} & 0 & 0 \\
0 & 0 & \alpha_{6} & \alpha_{4} & 0 \\
0 & 0 & \alpha_{7} & 0 & \alpha_{4}
\end{array}\right)
$$

where the $\alpha_{i}$ are taken from a field $F$. Then the set of matrices of the form (19) with all but the first column zero is a dominant left ideal of $A$ and hence, $A$ is a dominant algebra. On the other hand, the set of matrices of the form (19) with all but the third column zero form a primitive left ideal of $A$ which is neither dominant nor weakly subordinate to any set of dominant ideals of $A$. Therefore $A$ is not a $Q F-3$ algebra.

In studying an algebra it is often useful to study the residue class algebra $A / N^{i}$ where $N$ is the radical of $A$. Since imposing conditions on all of the $A / N^{i}$ often imposes very strong conditions on $A$ itself, it might be conjectured that requiring that each $A / N^{i}$ be dominant might be sufficient to imply that $A$ is generalised uniserial. However, this is false, as is shown by the example (19). The radical of $A$ in this case, is the set of all matrices of the form in (20)

$$
\left(\begin{array}{lllll}
0 & 0 & 0 & 0 & 0 \\
\alpha_{5} & 0 & 0 & 0 & 0 \\
0 & 0 & 0 & 0 & 0 \\
0 & 0 & \alpha_{6} & 0 & 0 \\
0 & 0 & \alpha_{7} & 0 & 0
\end{array}\right)
$$


and $N^{2}=0$. But $A / N$ and $A / N^{2}$ are both dominant but $A$ is not generalised uniserial; the primitive ideal composed of the matrices with all columns zero but the third has more than one composition series.

Thus, in particular, the result (3, Lemma) that a $Q F-3$ algebra $A$ with $N^{2}=0$ is generalised uniserial cannot be generalised by replacing the $Q F-3$ condition by one assuming that $A$ is dominant.

\section{REFERENCES}

(1) Emil Artin, C. J. Nesbitt and R. M. Thrall, Rings with minimum condition, Univ. of Michigan Publications in Math., No. 1, 1944.

(2) Nathan Jacobson, Structure of Rings (Amer. Math. Soc. Colloquium Publication No. 37, New York, 1956).

(3) YutaKa Kawada, A generalisation of Morita's theorem concerning generalised uniserial algebras, Proc. Japan Acad. 34 (1958), 404-406.

(4) Kirt Morita, Duality for modules, Sci. Rep. Tokyo Kyoiku Daigaku, 6, no. 150 (1958), 83-142.

(5) R. M. Thrall, Some generalisations of quasi-Frobenius algebras, Trans. Amer. Math. Soc., 64 (1948), 173-183.

(6) DRURY W. WALL, Characterisations of generalised uniserial algebras. I, Trans. Amer. Math. Soc., 90 (1959), 161-170.

(7) DruRy W. WALL, Characterisations of generalised uniserial algebras. II, Proc. Amer. Math. Soc., 9 (1958), 915-919.

(8) DruRy W. Wall, Algebras with unique minimal faithful representations, Duke Math., J. 25 (1958), 321-330.

(9) DruRY W. WALL, Cartan invariants of algebras with unique minimal faithful representations, Illinois $J$. Math., 4 (1960), 133-142.

THE UNIVERSITY OF IOWA

IOWA CitY, Iowa 